\title{
Long noncoding RNA PANDAR blocks CDKN1A gene transcription by competitive interaction with p53 protein in gastric cancer
}

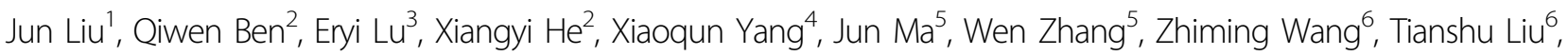 \\ Jianjun Zhang ${ }^{7}$ and Hongxia Wang ${ }^{1}$
}

\begin{abstract}
Emerging evidence indicates that IncRNAs play important roles in cancer tumourigenesis and could be used as potential diagnostic biomarkers or therapeutic targets. However, the clinical significance and molecular mechanism of IncRNAs in gastric cancer (GC) is still unclear. The aim of this study was to explore the expression and role of IncRNAs in GC. The relative expression level of IncRNAs in GC samples was examined by an IncRNA microarray analysis, northern blot analysis and qRT-PCR analysis. A Kaplan-Meier survival analysis and univariate and multivariate Cox proportional hazards models were performed to evaluate the clinical and prognostic significance of PANDAR (promoter of CDKN1A antisense DNA damage activated RNA) in GC patients. The binding activity of PANDAR with the p53 protein was analysed by an RNA immunoprecipitation analysis and RNA pull-down analysis. The depletion of PANDAR was conducted using the CRISPR/Cas9 system for PANDAR. The biological functions of PANDAR in GC cells were determined both in vitro and in vivo. Upregulated PANDAR in GC patients was positively correlated with increased tumour size, advanced TNM classification and a poor survival rate in GC patients. The ROC curves identified that the PANDAR level was a marker for discriminating the early-stage tumour group from the healthy group, the metastasis group from the non-metastasis group and the chemoresistance group from the chemosensitive group in GC patients. As a target, the CDKN1A gene was successfully downregulated by PANDAR. PANDAR controlled the transcription of the CDKN1A gene by competitively binding with p53 protein. In combination with a p53 activator (nutlin3), the knockout of PANDAR by CRISPR/Cas9 technology synergistically inhibited GC tumour growth in vivo. Our results suggest that the PANDAR is a powerful diagnostic and therapeutic marker for patients with GC and, combined with other chemotherapeutics, may have distinct antitumour effects.
\end{abstract}

Correspondence: Tianshu Liu (liu.tianshu@zs-hospital.sh.cn) or Jianjun Zhang (zjjshuobo@163.com) or Hongxia Wang (whx365@126.com) 'Department of Oncology, Shanghai General Hospital, Shanghai Jiao Tong University School of Medicine, 200080 Shanghai, PR China

2Department of Gastroenterology, Ruijin Hospital, Shanghai Jiao Tong

University School of Medicine, 200025 Shanghai, PR China

Full list of author information is available at the end of the article

Jun Liu and Qiwen Ben are contributed equally to this work.

Edited by A. Stephanou

\section{Introduction}

Gastric cancer (GC) is one of the most common cancers and accounts for a notable proportion of global cancer mortality ${ }^{1}$. Gastric cancer is often diagnosed at an advanced stage in the majority of patients and is associated with poor 5-year survival rates ${ }^{2}$. Despite recent advances in medical treatment, there has been little improvement in the early diagnosis and treatment of 
$\mathrm{GC}^{3,4}$. Increasing evidence indicates that long noncoding RNAs (lncRNAs) play critical roles in a wide range of biological processes, including cell development, differentiation, immune responses and tumourigenesis ${ }^{5,6}$. Understanding the contributions of lncRNAs to GC progression will offer insights into tumour transformation and help to identify new biomarkers and novel treatment targets for this disease.

LncRNAs refer to RNAs that lack coding potential, are $>200$ bp in length and are pervasively transcribed in the genome $^{7}$. LncRNAs play critical roles as tumour suppressors or oncogenes by activating or silencing the expression of protein-coding genes ${ }^{8}$. The lncRNA HOTAIR induces the genome-wide re-targeting of Polycomb Repressive Complex 2 and leads to altered histone H3 lysine 27 methylation and increased breast cancer metastasis $^{9-11}$. The IncRNA MALAT1 is required for G1/ $\mathrm{S}$ and mitotic progression, and $\mathrm{p} 53$ is a major downstream mediator of MALAT1 activity ${ }^{12}$. For GC, the upregulation of HOTAIR is associated with more venous invasion, frequent lymph node metastases and a lower overall survival rate $^{13}$. The overexpression of the lncRNA H19 dramatically promotes GC cell proliferation and metastasis by the direct upregulation of the ISM1 protein ${ }^{14}$. The lncRNA GAPLINC is highly expressed in GC tissues and mainly changes the migratory ability of the cancer cells by altering the levels of CD44 mRNA ${ }^{15}$. Low lncRNA GAS5 expression in GC is associated with poorer overall survival, and the ectopic expression of GAS5 influences GC cell proliferation via regulating E2F1 and CDKN1A expression ${ }^{16}$. Acting as a competing endogenous RNA, lncRNA-FER1L4 regulates the expression of PTEN, CDKN1A and E2F1 through its miRNA response elements to compete for miR-106a-5 $p^{17}$. The lncRNA LEIGC suppresses tumour growth and enhances the sensitivity of GC cells to 5 -fluorouracil by inhibiting the epithelial-to-mesenchymal transition ${ }^{18}$. Therefore, deeply understanding the roles of IncRNAs in tumourigenesis has an important significance in the development of molecular-targeted therapy.

In this study, we report findings implicating the lncRNA PANDAR (promoter of CDKN1A antisense DNA damage activated RNA) (LNCipedia.org; Gene ID: PANDAR) in GC based on the use of global microarray analyses in human GC specimens. We analysed the relationship between PANDAR levels and the clinicopathological features of GC, including clinical outcome. We deeply investigated the biological effect and mechanisms of altered PANDAR levels on the phenotypes of GC cells in vitro and in vivo. Our findings suggest that PANDAR may represent a novel indicator of poor prognosis in GC and may be a potential diagnostic and therapeutic marker.

\section{Materials and methods}

\section{Patients and reagents}

Between April 2007 and April 2008, we recruited 146 patients with primary GC from the Zhongshan hospital and Shanghai Cancer Center, Fudan University. All the patients (excluding the stage IV disease) underwent an R0 resection (with no tumour cells at the margin) with D2 lymph node dissection, and all the patients histopathologically confirmed for the diagnosis of stages II and III received 6-8 courses of chemotherapy-based fluorouracil following the operation. The Ethics Committee of Fudan University approved our study. All the participants provided written informed consent prior to enrolment. The human GC cell lines were obtained from the American Type Culture Collection (Manassas, VA, USA) and were identified through an STR analysis. The p53 antibody (\#9282) and p21 antibody (\#8831) were purchased from Cell Signaling Technology (Beverly, MA, USA). Nutlin-3 (S1061) was purchased from Selleck (Princeton, NJ, USA).

\section{CRISPR/Cas9-mediated deletion of PANDAR/TP53/CDKN1A}

The CRISPR (clustered regularly interspaced short palindromic repeats)/Cas9 system has the ability to infect a broad variety of mammalian cells by co-expressing a mammalian codon-optimized Cas9 nuclease along with a single guide RNA (sgRNA) to facilitate genome editing. The lentiCRISPRv2 (Plasmid \#52961)) was obtained from Addgene (Cambridge, MA, USA). The lentiCRISPRv2 plasmid contains two expression cassettes, hSpCas9 and the chimeric guide RNA. The vector was first digested using BsmBI (\#R0580S, NEB, Beijing, China), and a pair of annealed oligos was cloned into the single guide RNA scaffold. The oligos were designed based on the target site sequence and were flanked on the $3^{\prime}$ end by a $3 \mathrm{bp}$ NGG PAM sequence. The sgRNA sequence for PANDAR was designed using the CCTop-CRISPR/Cas9 target online predictor (http://crispr.cos.uni-heidelberg.de/ index.html). The sgRNA sequences for TP53 and CDKN1A were designed using E-CRISP-Version 5.2 website (http://www.e-crisp.org/E-CRISP/). After $72 \mathrm{~h}$ of infection with the lentiCRISPRv2 plasmid, the single cell clones were selected by puromycin at a concentration of $10 \mu \mathrm{g} / \mathrm{ml}$.

\section{LncRNA microarray analysis}

The lncRNA expression profiles were investigated using the Agilent Human lncRNA array 8*60K (OE Biotech, Shanghai, China). The RNA samples were first reverse transcribed into cDNA, and these cDNA samples were then labelled using the Low Input Quick-Amp Labeling Kit (Agilent Technologies, Santa Clara, CA, USA). The labelled cDNA samples were used as probes to hybridize 
to the lncRNA microarrays. After the samples were hybridized, the microarrays were scanned with an Agilent microarray scanner. Feature Extraction software (version 10.7.1.1, Agilent Technologies) was used to analyse the array images to obtain the raw data. Gene-Spring software (version 12.5, Agilent Technologies) was employed to finish the basic analysis of the raw data. Initially, the raw data were normalized using the quantile algorithm. The probes with at least $100 \%$ of the samples in any one condition out of two conditions having flags that indicated 'Detected' were chosen for further data analysis. The differentially expressed lncRNAs were then identified through the fold change, and $p$ values were calculated using $t$-tests. The thresholds set for the up- and downregulated genes were a fold change $\geq 2.0$ and a $p$ value $\leq$ 0.05 .

These microarray data were approved and were assigned the GEO accession number GSE84787.

\section{Northern blot analysis}

PANDAR levels were measured by northern blotting using an Ambion Northern Max-Gly Kit (Austin, TX, USA). The total RNA was electrophoresed on a $1 \%$ agarose gel and was siphoned to a positively charged nylon membrane (NC). The RNA was then fixed to the NC membrane using UV crosslinking. The cross-linked membrane was prehybridized with ultrahyb-oligo hybridization buffer and hybridized with the PANDAR-specific oligonucleotide probes (AACATTGGGTGGGGCGAGTCAT) labelled with digoxigenin-ddUTP in roller bottles. The probe was designed using the EXIQON website (https://www.exiqon.com).

\section{Plasmid construction}

Full-length sequences of PANDAR and the wild-type TP53 gene were amplified by PCR. AGS cells were used as the PCR template. The PCR products were first subcloned into the $\mathrm{T}$ vector and were then cloned into the pLKO.1 plasmid (Addgene, Cambridge, MA, USA). We used the Phusion Site-Directed Mutagenesis Kit (Life Technologies, Grand Island, NY, USA) to construct the TP53 mutated vector. For lentiviral vector packaging, three plasmids are transfected into 293T cells, including the transfer vector $(3 \mu \mathrm{g})$, delta $8.9(3 \mu \mathrm{g})$ and VSV-G $(1$ $\mu \mathrm{g})$. After a media change overnight, the supernatant containing the virus was stored.

\section{QRT-PCR analysis}

Total RNA was extracted from GC tissues and cell lines using the TRIzol Reagent (Invitrogen, Shanghai, China). For the reverse transcript reaction, $1 \mu \mathrm{g}$ of total RNA was used and was mixed with the random primer. The reaction was carried out for $30 \mathrm{~min}$ at $16^{\circ} \mathrm{C}, 30 \mathrm{~min}$ at $42^{\circ} \mathrm{C}$, and $5 \mathrm{~min}$ at $85^{\circ} \mathrm{C}$ and was then held at $4{ }^{\circ} \mathrm{C}$. QRT-PCR was performed to measure PANDAR expression levels using the PowerUp ${ }^{\mathrm{TM}} \mathrm{SYBR}^{\circ}$ Green Master Mix (Life Technologies, Grand Island, NY, USA). The reaction conditions were as follows: 1 cycle of $95^{\circ} \mathrm{C}$ for $1 \mathrm{~m} ; 38$ cycles of $95^{\circ} \mathrm{C}$ for $5 \mathrm{~s}$ and $65^{\circ} \mathrm{C}$ for $30 \mathrm{~s}$. We used the delta delta CT method to calculate the fold change in gene expression between the different groups. The primers used for these analyses are listed in Supplementary Table 4.

\section{Immunoblotting analysis}

The whole cell lysates $(50 \mu \mathrm{g})$ were electrophoresed using $10 \%$ polyacrylamide gels and were transferred to a PVDF membrane. The membrane was incubated with the p53 antibody (1:1000), p21 antibody (1:1000) or HA mouse antibody (1:3000). The secondary antibodies (1:3000) were labelled with horseradish peroxidase (HRP). The signals were checked using an autoradiography film when the HRP substrate was added to the membrane.

\section{ChIP analysis}

The CHIP protocol was described in detail in a previous study $^{19}$. The proteins were cross-linked to the DNA by adding formaldehyde at room temperature for $10 \mathrm{~min}$, and the DNA was sheared to an average fragment size of $300-500$ bp by sonication. Subsequently, immunoprecipitation was performed using the p53 antibody. The purified DNA was quantified by qRT-PCR using the PowerUp ${ }^{\mathrm{TM}}$ SYBR ${ }^{\circ}$ Green Master Mix (Life Technologies, Grand Island, NY, USA). The primer pairs used for analysis are described in Supplementary Table 4.

\section{RNA immunoprecipitation (RIP) analysis}

GC cells were treated with the appropriate vectors for $48 \mathrm{~h}$ and were harvested by trypsinization. The nuclei were pelleted by centrifugation, were re-suspended using Magna Nuclear RIP ${ }^{\mathrm{TM}}$ Kits (Millipore, Billerica, MA, USA) and were mechanically sheared using a homogenizer. The HA antibody was added to the nucleic extraction, and it was incubated overnight at $4{ }^{\circ} \mathrm{C}$. The $\mathrm{HA}$ beads were then added and incubated for $4 \mathrm{~h}$ at $4{ }^{\circ} \mathrm{C}$. The HA beads were pelleted, washed and re-suspended in $1 \mathrm{ml}$ of TRIzol. The isolated RNA was reverse transcribed to cDNA and then analysed with qRT-PCR.

\section{RNA pull-down analysis}

The RNA pull-down analysis protocol was described in detail in a previous study ${ }^{20}$. The biotinylated PANDAR and U6 RNA were mixed with proteins from the nuclear extract of the cancer cells. The complex of the biotinylated PANDAR and proteins was purified using streptavidin-agarose. The proteins were then eluted from the RNA-protein complex and detected by immunoblotting using the p53 antibody or HA antibody. 

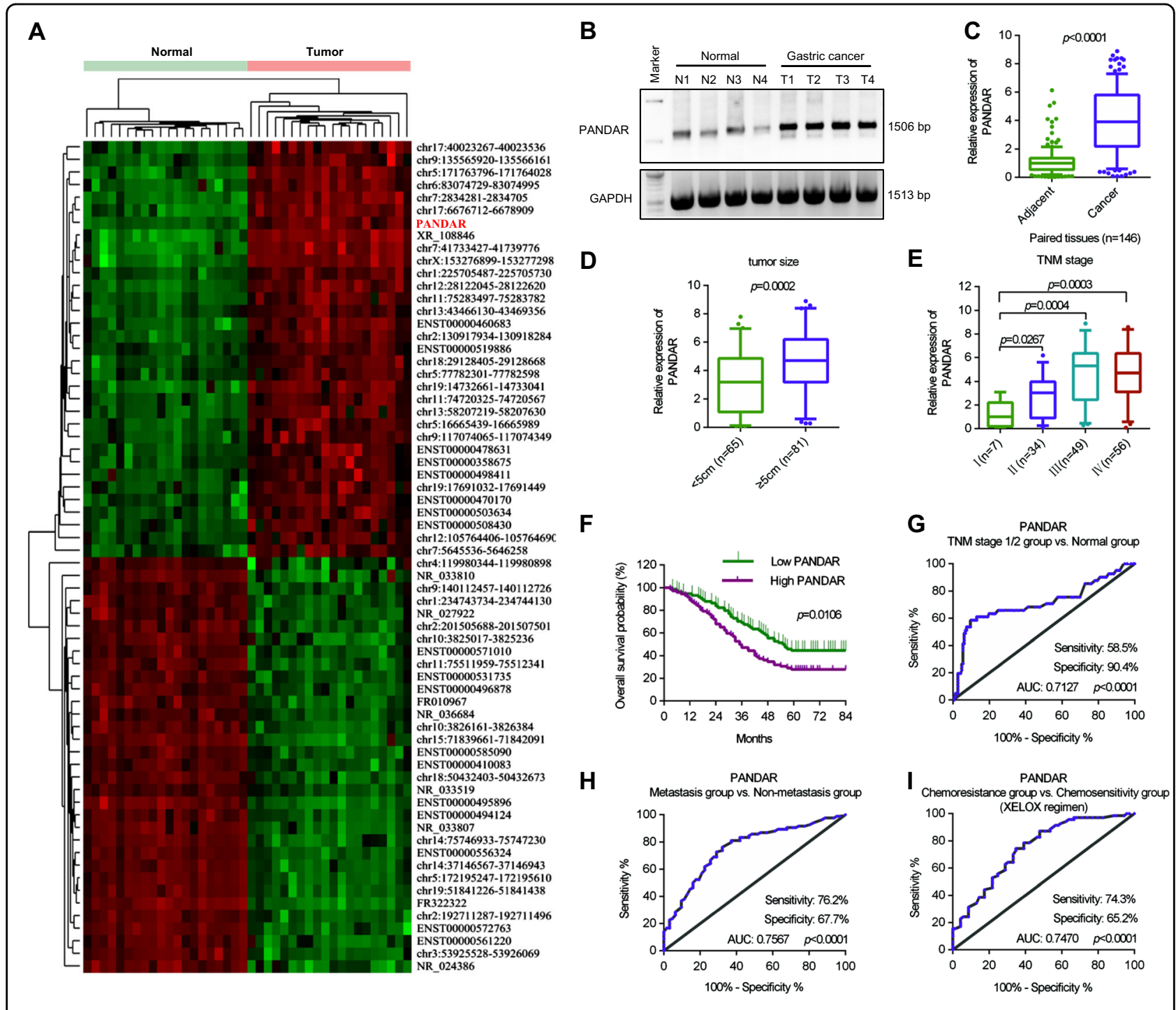

Fig. 1 Prognostic and diagnostic significance of PANDAR for GC patients. a LnCRNA expression profiles were evaluated by an IncRNA expression microarray. Heat map showed different IncRNA expression among samples. 'Red' indicates high expression; 'Green' indicates low expression. b The expression of PANDAR was demonstrated using northern blotting analysis in paired GC samples. c The expression of PANDAR was demonstrated using real-time PCR analysis in paired GC samples. $\mathbf{d}$ High levels of PANDAR in GC tissues correlated with increased tumour size. e High levels of PANDAR in GC tissues correlated with increased TNM stage. $\mathbf{f}$ Kaplan-Meier analyses of the overall survival. Patients with a high level of PANDAR expression (above mean; $n=72$ ) were associated with a significantly lower overall survival rate than patients with a low level of PANDAR expression (below mean; $n=74$ ). $\mathbf{g}$ ROC plots for the PANDAR discriminating the TNM stage $1 / 2$ group from the healthy group. AUC area under the curve. $\mathbf{h}$ ROC plots for the PANDAR discriminating the metastasis group from the non-metastasis group. $\mathbf{i}$ ROC plots for the PANDAR discriminating the chemoresistance group from the chemosensitivity group. XELOX regimen: Capecitabine $825-1000 \mathrm{mg} / \mathrm{m}^{2}$ bid po d1 $1-14$ q3w; Oxaliplatin $130 \mathrm{mg} / \mathrm{m}^{2}$ ivgtt d1 q3w

\section{Cell proliferation and clone formation analysis}

AGS cells and SNU-1 cells were transfected with the lenti-PANDAR vector or the knockout of PANDAR for $48 \mathrm{~h}$ and were seeded onto 96-well plates (1000 cells/per well) and $10 \mathrm{~cm}$ dishes (1000 cells/per well). Cell viability was measured daily using the CCK- 8 assay (Dojindo, Shanghai, China). The clone formation ability was determined after 2 weeks of culture by crystal violet staining.

\section{Immunohistochemistry analysis}

The mouse tumour tissues were fixed in formalin, embedded in paraffin, spliced into $6 \mu \mathrm{m}$ sections, deparaffinized with xylene and submerged into EDTA antigenic retrieval buffer for antigenic retrieval. The tumour sections were incubated with the p21 antibody overnight at 4 ${ }^{\circ} \mathrm{C}$, treated with an anti-rabbit secondary antibody for $1 \mathrm{~h}$ at RT and incubated with the streptavidin-horseradish 
peroxidase complex. The sections were stained with haematoxylin.

\section{In vivo analysis}

Stable PANDAR-depleted AGS cells $\left(1 \times 10^{6}\right)$ were subcutaneously injected into the right flank of 3-week-old athymic nude mice $(n=6)$. The xenografts were treated with nutlin-3 via an oral administration at $200 \mathrm{mg} / \mathrm{kg}$ once daily for 6 weeks. The nude mice were killed after 6 weeks of nutlin- 3 treatment, and the tumour weight and inhibitory rate were measured and calculated.

\section{Optical in vivo imaging analysis}

Stable PANDAR-depleted AGS cells $\left(1 \times 10^{6}\right)$ were injected into immunodeficient mice ( 3 animals per group) by tail vein assays and subsequently received $200 \mathrm{mg} / \mathrm{kg}$ of an oral dose of nutlin-3 once daily. After 2 weeks, the nude mice were evaluated for lung colonization capacity. Tumour progression was monitored by imaging with a Xenogen Spectrum small animal imaging system 15 min after an injection of $100 \mu \mathrm{l}$ Luciferin Substrate (Caliper, Hopkinton, MA) IP into the mice.

\section{Statistical analyses}

All the data are expressed as the mean \pm standard deviation. Two variables of the microarray data and qRTPCR data were analysed using Student's $t$-test. The categorical variables were analysed using the chi-square $\left(\chi^{2}\right)$ test. The 5-year survival rate analyses of GC patients were performed using the log-rank (Mantel-Cox) test. The tumour weights were evaluated by Mann-Whitney $\mathrm{t}$ test. A $p$ value of $<0.05$ was considered significant.

\section{Results \\ Overexpression of PANDAR in GC patients indicates malignant transformation}

We compared the IncRNA expression profiles of the GC tissues and adjacent normal tissues using unsupervised hierarchical clustering in 20 patients (Fig. 1a). We identified the top ten upregulated lncRNAs using qRT-PCR in 20 paired GC samples. As shown in Supplementary Figure 1A, the expression levels of the selected lncRNAs were identified using both study analyses. PANDAR was the most changed lncRNA among the upregulated lncRNAs. Compared with the normal samples, the upregulation of PANDAR was identified by a northern blotting analysis in the paired tumour tissues (Fig. 1b). We validated the expression level of PANDAR by a qRT-PCR analysis in 146 paired GC samples (Fig. 1c). The expression of PANDAR was closely associated with tumour size, TNM classification and N classification (Figs. 1d, e; Supplementary Table 2). Gastric cancer patients with a high expression of PANDAR showed significantly shorter 5year overall survival rates than those with low expression of PANDAR (Fig. 1f). In the univariate and multivariable analysis, PANDAR expression, TNM classification and $\mathrm{N}$ classification were significant independent prognostic factors for survival time (Supplementary Table 3). Receiver operating characteristics (ROC) curves were constructed to evaluate the performance of PANDAR as a marker for discriminating the early-stage group from the healthy group (Fig. 1g), the metastasis group from the non-metastasis group (Fig. 1h) and the chemoresistance group from the chemosensitive group (Fig. 1i) in GC patients. These results revealed that PANDAR mRNA levels in GC tissues might be a promising prognostic (metastasis and chemoresistance) and diagnostic (early diagnosis) indicator.

\section{PANDAR promotes the malignant progression of GC cells}

We investigated the expression level of PANDAR by a northern blotting analysis and the qRT-PCR method in normal gastric tissues and in ten GC cell lines. Compared with the normal samples, the upregulation of PANDAR was identified in the majority of GC cell lines except for the NCI-N87 and SNU-1 cells (Fig. 2a, b). The demethylation of the PANDAR promoter may be responsible for PANDAR overexpression in GC tissues (Fig. 2c). Using the CRISPR/Cas9 system, the expression level of PANDAR was dramatically knocked out in AGS cells (Supplementary Figure 1B). The depletion of PANDAR obviously suppressed the proliferative and clone-forming activity of the AGS cells (Fig. 2d, e). Using flow cytometry, we found that PANDAR knockout blocked the cell-cycle progression at the G1/S checkpoint (Fig. 2f). We also identified that the depletion of PANDAR statistically promoted apoptotic activity in the AGS cells (Fig. 2g). In contrast, the exogenous expression of PANDAR, using a lentiviral expression vector (lenti-PANDAR), significantly increased the proliferation and clone-forming ability of SNU-1 cells (Supplementary Figure 1C; Fig. 2h, i). Furthermore, the overexpression of PANDAR promoted $\mathrm{S}$ phase entry (Fig. 2j) and suppressed the cancer cell apoptotic activity (Fig. 2k). To determine the relationship between PANDAR and the chemotherapy drug oxaliplatin, we identified the cell cytotoxicity of oxaliplatin in the cancer cells. The sensitivity of PANDAR-depleted AGS cells to oxaliplatin increased. In contrast, the sensitivity of PANDAR-overexpressed SNU-1 cells to oxaliplatin decreased. These results revealed that the knockout of PANDAR led to an increased sensitivity of GC cells to oxaliplatin (Supplementary Figure 2).

\section{PANDAR regulates CDKN1A gene transcription in a p53- dependent manner}

To identify the functional putative targets of PANDAR, we investigated the gene expression profiles using a microarrays analysis in the PANDAR-depleted AGS cells. 


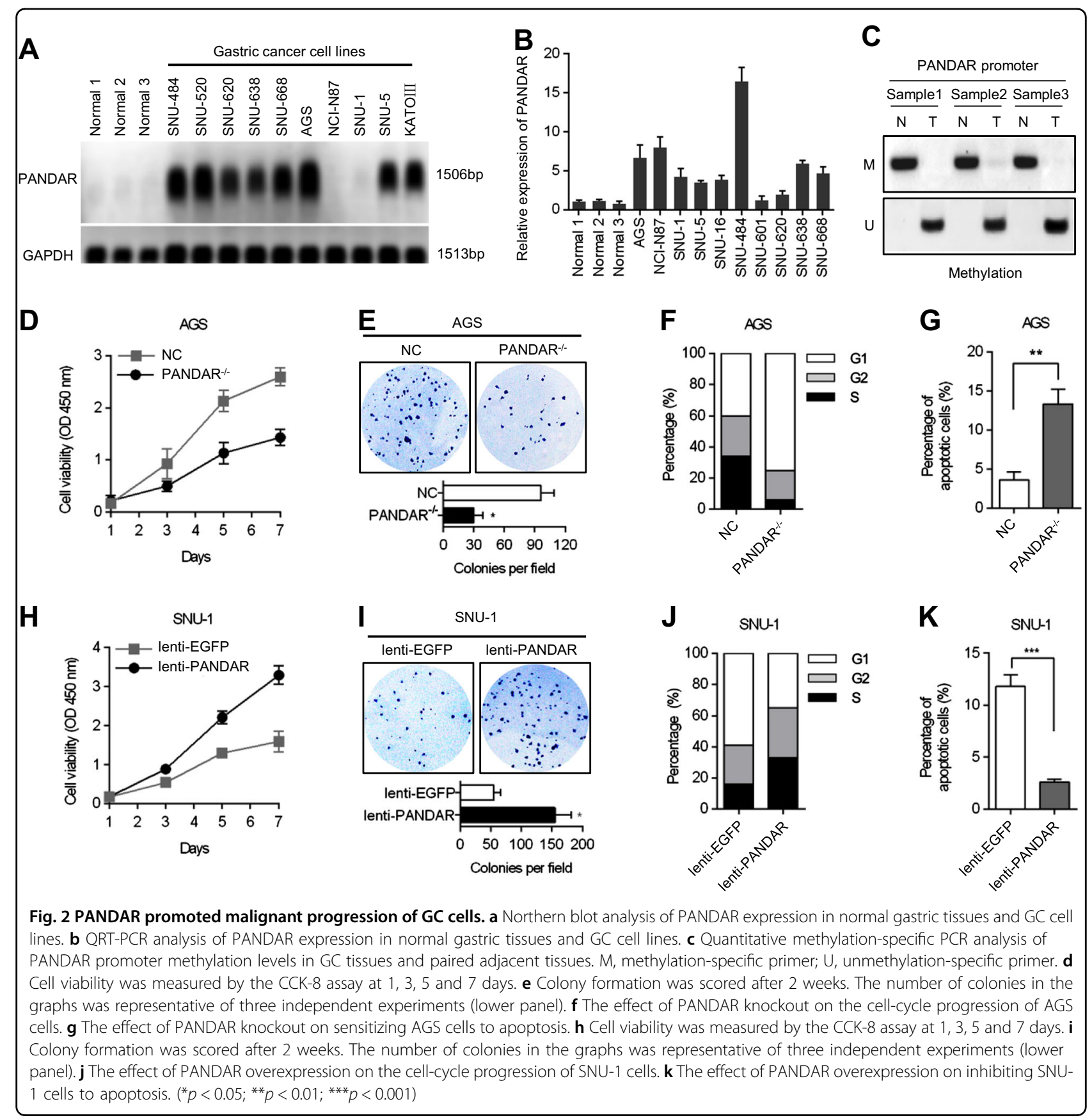

Compared with the negative control groups, 69 genes were upregulated and 17 genes were downregulated (Supplementary Figure 3). In contrast, we also investigated the gene expression profiles using a microarrays analysis in the AGS cells transfected with the lentiPANDAR. Twenty-eight genes were upregulated and 75 genes were downregulated in the PANDAR overexpression groups (Supplementary Figure 4). To reduce the false-positive rate, we intersected the gene expression profiles obtained from both of the above analyses. As shown in Fig. 3a, 18 putative PANDAR targets were identified and participated in multiple biological processes. The intersection results also implied the inhibitory transcription activity for PANDAR due to only two genes being found in the opposite intersection group (Supplementary Figure 5). We then determined the expression of these candidates using qRT-PCR in the PANDARdepleted AGS cells, and we identified that the most changed gene was $C D K N 1 A$, which is a key cell cycle checkpoint gene (Fig. 3b). 


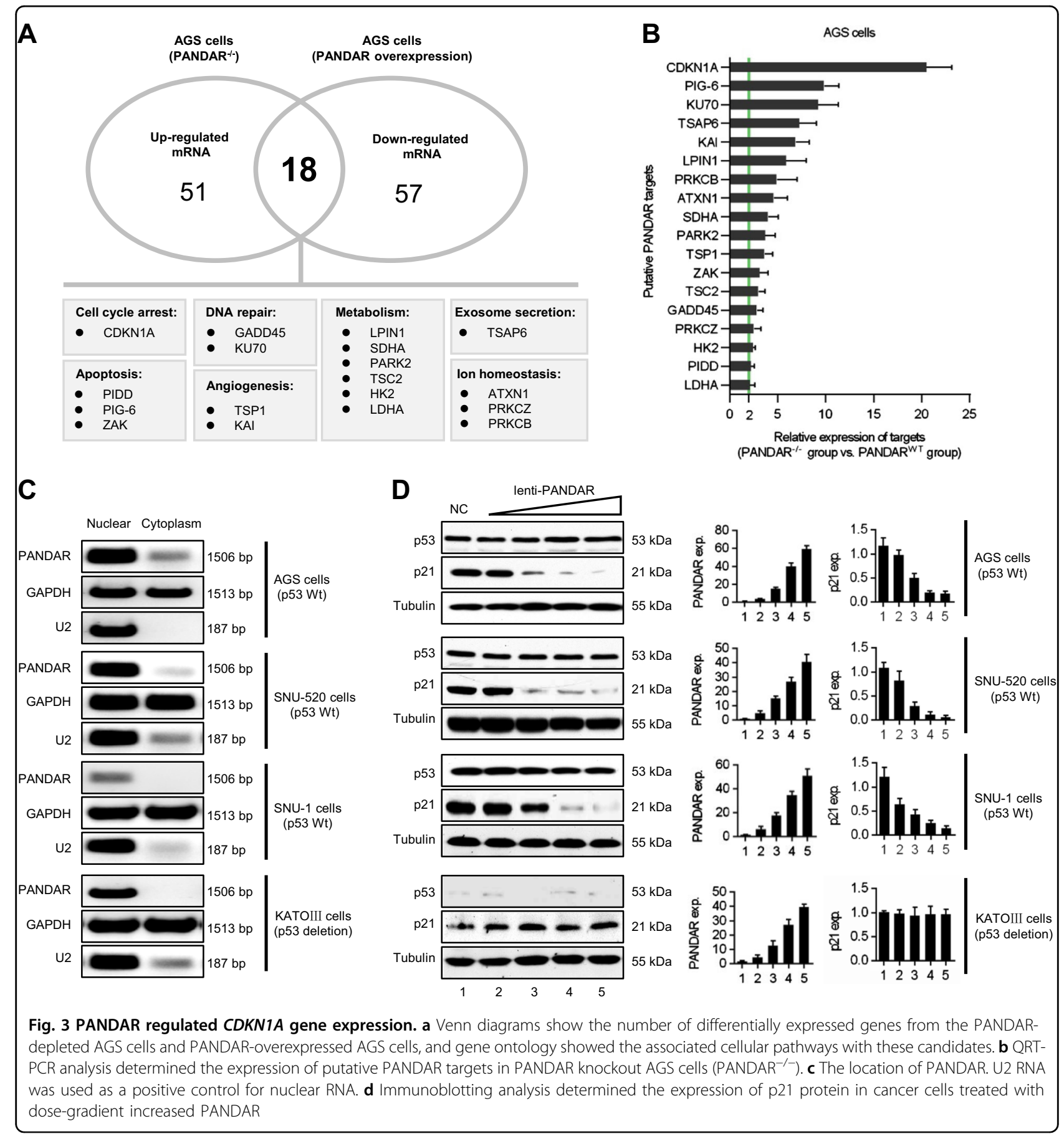

By isolating both nuclear and cytoplasmic RNA, we showed that PANDAR was mainly present in the nucleus (Fig. 3c). Our results showed that p21 levels were downregulated by PANDAR in a dose-dependent manner in p53 wild-type cancer cells but not in the p53 deletion mutation cancer cells (Fig. 3d). These results indicated that PANDAR regulates CDKN1A gene transcription in a p53-dependent manner.
PANDAR disturbs the binding of the p53 protein with the CDKN1A promoter

We conducted a competition assay to investigate the interaction of $\mathrm{p} 53$ protein and PANDAR. The biotinlabelled CDKN1A promoter $(1 \mu \mathrm{g})$ was synthesized and incubated with the purified p53 protein. We then added purified PANDAR produced by RNA synthesis to the reaction mixture (Fig. 4a). Before the assay, we evaluated 


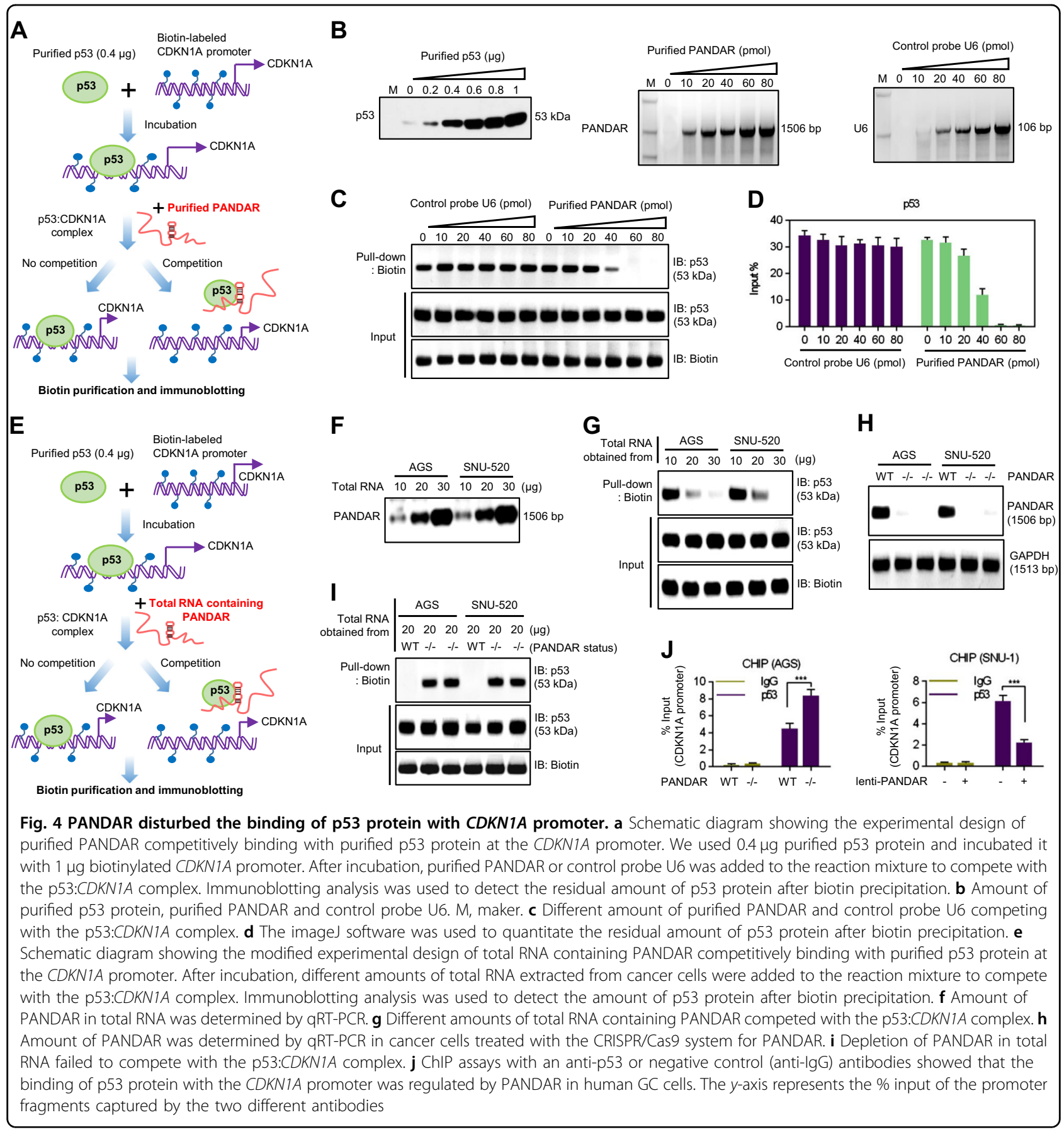

the amount of purified p53 protein, purified PANDAR and control probe U6 used in this examination (Fig. 4b). After adding the variant amount of purified PANDAR or control probe U6, we found that PANDAR significantly abolished the binding of $\mathrm{p} 53$ protein with the CDKN1A promoter in the presence of $40 \mathrm{pmol}$ of purified PANDAR (Fig. 4c). We also confirmed the results in quantitative assay (Fig. 4d).

Next, we modified the experimental design to determine whether the endogenic PANDAR in the cancer cells interacted with the p53 protein. The total RNA extracted from the AGS and SNU-520 cells was used instead of the purified PANDAR (Fig. 4e). We identified that $20 \mu \mathrm{g}$ of total cellular RNA containing PANDAR successfully abrogated the binding of the p53 protein with the CDKN1A promoter in the AGS and SNU-520 cells (Fig. 4f, g). After PANDAR knockout (Fig. 4h), the p53 protein maintained its interaction with the CDKN1A promoter to form the p53:CDKN1A complex (Fig. 4i). We also performed chromatin immunoprecipitation 


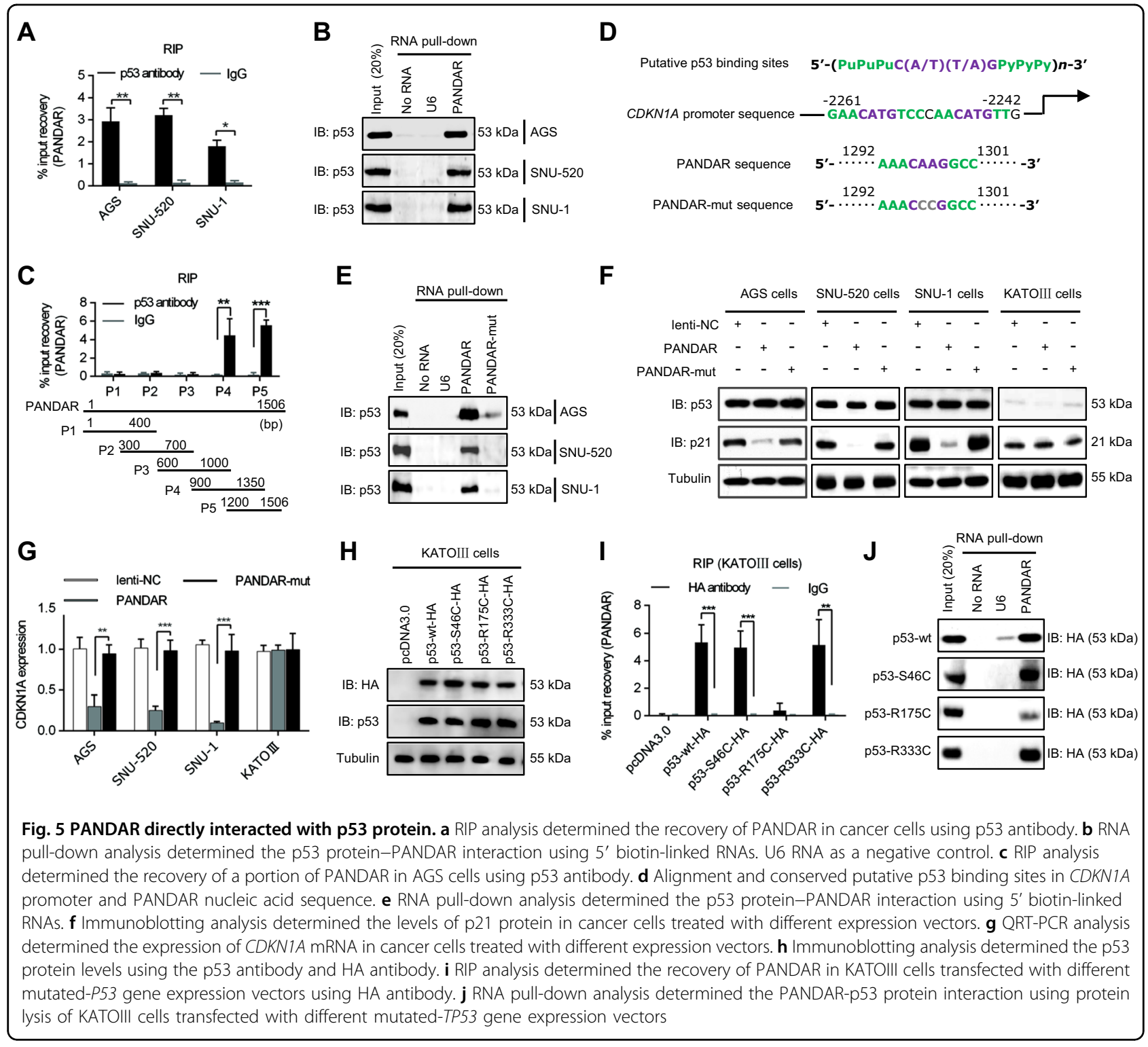

experiments to determine the influence of PANDAR on the p53:CDKN1A promoter complex. We defined the upstream 3000 bases of the first exon of the CDKN1A gene as the CDKN1A promoter. As shown in Fig. $4 \mathrm{j}$, the depletion of PANDAR increased the binding of the p53 protein to the CDKN1A promoter. However, the overexpression of PANDAR decreased the binding of the p53 protein to the CDKN1A promoter.

\section{PANDAR directly interacts with the $\mathrm{p} 53$ protein}

To investigate whether the biological effect of PANDAR was related to its binding ability with $\mathrm{p} 53$ protein, we performed RNA immunoprecipitation (RIP) experiments with p53 wild-type cancer cells (AGS, SNU-520 and SNU1) (Fig. 5a). Our results clearly showed that PANDAR was obviously recovered with the p53 protein. In contrast, we performed an RNA pull-down assay using various species of $5^{\prime}$ biotin-linked RNAs. As shown in Fig. 5b, the p53 protein was only pulled down by PANDAR but not by the negative control (U6). To investigate the region of PANDAR responsible for the binding activity of the p53 protein, we designed various primers to detect the different fragments of PANDAR. Both part 4 (P4) and part 5 (P5) of PANDAR were markedly recovered by the p53 protein (Fig. 5c). In further analyses, we identified a nucleotide sequence which might be responsible for binding with the p53 protein in PANDAR (Fig. 5d). After mutation at this p53 putative binding site, the p53 protein could not be effectively pulled down by PANDAR (Fig. 5e). The PANDAR mutation also abrogated its competitive inhibitory ability towards the $\mathrm{p} 53$ protein in the $\mathrm{p} 53$ wild-type cancer cells (AGS, SNU-520 and SNU-1) (Fig. 5f). We also 


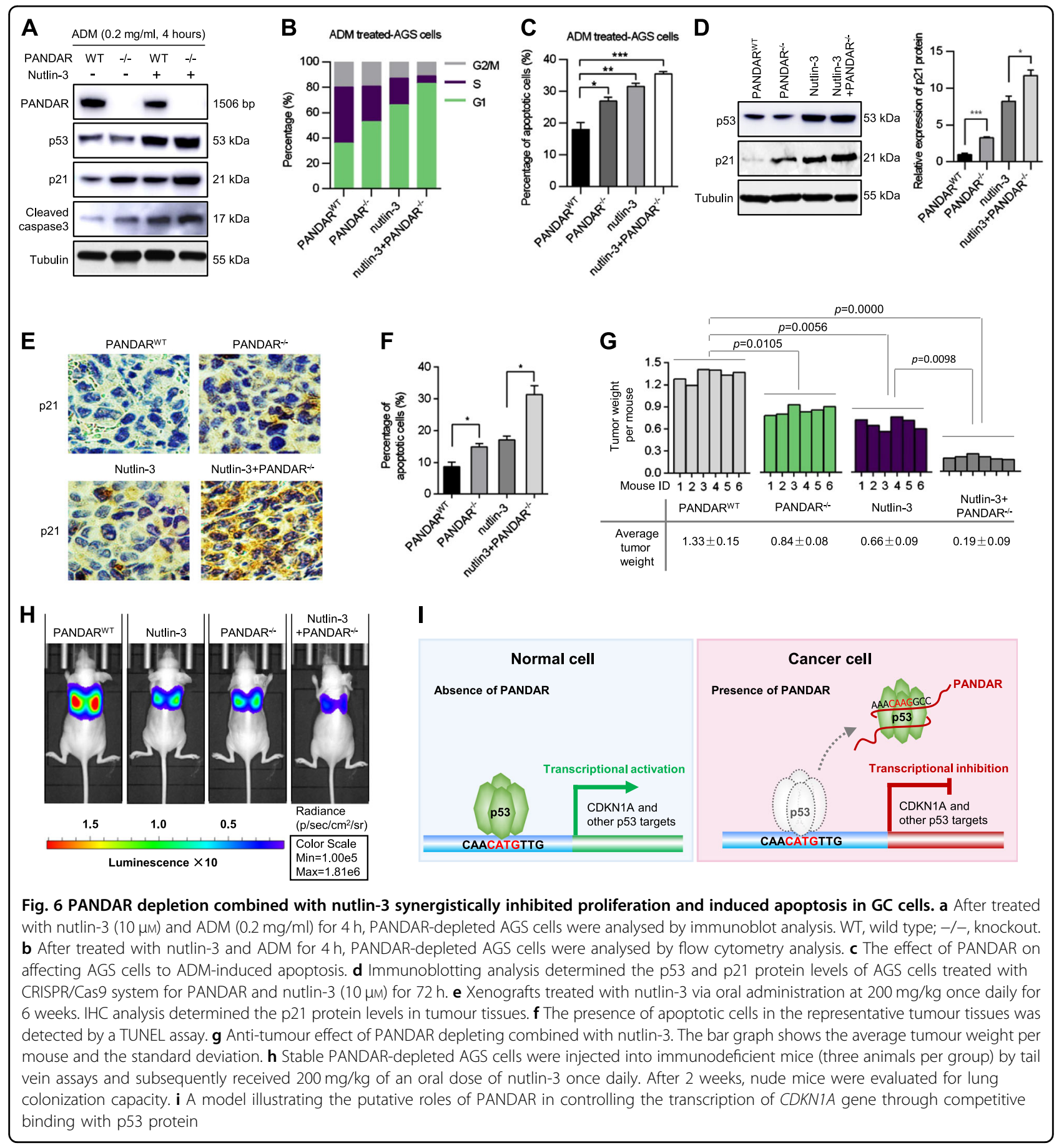

showed that the overexpression of PANDAR reduced the expression of CDKN1A, whereas the overexpression of mutant PANDAR did not affect the CDKN1A expression at the mRNA level (Fig. 5g). These results indicated that PANDAR interacted with the p53 protein through its 1292-1301 bp nucleic acid sequence (aaCAAGgcc).

To investigate which motif of p53 protein was responsible for the binding activity towards PANDAR, we constructed a series of TP53-mutated expression vectors. We first determined the protein expression levels of these TP53-mutated expression vectors in KATOIII cells (Fig. 5h). Then, we performed an RIP analysis with the KATOIII cells (p53 deletion) transfected with different TP53-mutated expression vectors using the HA-antibody (Fig. 5i). Our results showed that the DNA-binding domain mutation (R175C) suppressed the recovery of 
PANDAR with the p53 protein. Additionally, using $5^{\prime}$ biotin-linked RNAs, we performed an RNA pull-down analysis to define the binding activity of the mutated-p53 protein with PANDAR. The mutation at the DNAbinding domain of the p53 protein abrogated its ability to interact with PANDAR (Fig. 5j). These results illustrated that PANDAR inhibited CDKN1A gene transcription by competitively binding with the p53 protein.

\section{PANDAR depletion, combined with nutlin-3, inhibits proliferation and induces apoptosis in GC cells in vivo}

To determine whether PANDAR regulated the transcription of multiple targets mainly by interacting with the p53 protein, we performed a microarray analysis using the AGS cells in which p53 was overexpressed (Supplementary Figure 6). As shown in Supplementary Figure 7A, a large body of the PANDAR putative targets (53/69, 76.8\%) overlapped with the p53 targets. Furthermore, we confirmed the regulation of randomly selected p 53 targets by PANDAR knockout (Supplementary Figure 7B). We also performed a linear regression analysis for PANDAR and the randomly selected p53 targets in GC tissues (Supplementary Figure 7C). These results illustrated that PANDAR controlled the transcription of multiple targets mainly by interacting with $\mathrm{p} 53$.

To further identify the roles of CDKN1A and TP53 in tumour suppression, we first knocked out the CDKN1A gene or TP53 gene expression by the CRISPR/ Cas9 system. The depletion of the CDKN1A gene and TP53 gene dramatically promoted cancer cell growth (Supplementary Figure 8A). In addition, the upregulation of PANDAR lost their ability to promote tumour growth in the TP53-depleted AGS cells (Supplementary Figure $8 \mathrm{~B}$ ). These results indicated that PANDAR promoted tumourigenesis in a p53-dependent manner.

To observe whether the combined treatment of PANDAR depletion with the p53 activator nutlin-3 caused any changes in the stress response of the cells, we first used the CRISPR/Cas9 system to knock out PANDAR expression in the AGS cells (Supplementary Figure 8C). Then, the PANDAR-depleted AGS cells were treated with nutlin-3 and adriamycin (ADM) for $4 \mathrm{~h}$. The knockout of PANDAR combined with nutlin-3 dramatically increased p21 and cleaved caspase 3 protein expression in the ADMtreated AGS cells (Fig. 6a). Using flow cytometry, we found that the combination of PANDAR depletion and nutlin-3 blocked the cell-cycle progression at the G1/S checkpoint (Fig. 6b) and increased the apoptotic activity of the AGS cells (Fig. 6c).

We investigated whether PANDAR depletion combined with the p53 protein activator had a synergistic effect on cancer therapy. PANDAR depletion combined with nutlin-3 obviously promoted an increase in the p53 protein level and p21 protein level in the AGS cells (Fig. 6d).
After a subcutaneous injection of the PANDAR-depleted AGS cells and treatment with nutlin-3 for 6 weeks, the PANDAR depletion combined with nutlin-3 group showed increased levels of the p21 protein (Fig. 6e). The combination of PANDAR depletion and nutlin-3 increased AGS cell apoptosis (Fig. 6f). Additionally, the knockout of PANDAR combined with nutlin-3 reduced the tumour weight of the mice compared with the negative control-treated group (Fig. 6g). The PANDARdepleted AGS cells were injected into the tail vein of immunodeficient mice. The cell survival in the circulation and extravasation to and growth in the lungs were evaluated and analysed. After 2 weeks, we observed that the knockout of PANDAR combined with nutlin-3 remarkably inhibited the lung colonization capacity (Fig. 6h). These data indicated that a combination of PANDAR depletion and nutlin3 treatment has therapeutic potential in GC cells.

\section{Discussion}

PANDAR is a relatively novel lncRNA that plays an important role in the development of multiple cancers. Previous studies have reported that PANDAR was significantly upregulated in cholangiocarcinoma (CCA) patients ${ }^{21}$, bladder cancer patients ${ }^{22}$, clear cell renal cell carcinoma (ccRCC) patients ${ }^{23}$, hepatocellular carcinoma $^{24}$ and colorectal cancer patients ${ }^{25}$. The overexpression of PANDAR in cancer patients was closely associated with a higher histological grade, advanced TNM stage and poorer overall survival. High expression of PANDAR in GC patients was correlated with the depth of invasion, advanced TNM stage and lymphatic metastasis. Importantly, a high expression of PANDAR could serve as an independent unfavourable prognostic role in $\mathrm{GC}^{26}$.

In this study, we first investigated the different lncRNA expression patterns between GC tissues and paired adjacent normal tissues. A clinical pathology characteristic correlation analysis revealed that the upregulated PANDAR was associated with a statistically significant increased tumour size, TNM classification, N classification and a poor survival rate in GC patients. ROC curves identified that the PANDAR levels were a marker for discriminating the early-stage tumour group from the healthy group, the metastasis group from the nonmetastasis group and the chemoresistance group from the chemosensitive group in GC patients. Our results suggested that PANDAR is a new diagnostic and therapeutic marker for patients with GC.

Quite a few lncRNAs affect gene transcription via chromatin modification ${ }^{27,28}$, transcription machinery modulation $^{29}$, or specific transcription factor modula$\operatorname{tion}^{30}$, which implies that the dysregulated lncRNAs may have regulatory roles in cancer progression. PANDAR is 
induced in response to DNA damage and represses apoptosis by inhibiting the function of the transcription factor nuclear transcription factor $\mathrm{Y}$ subunit alpha (NFYA). Further experiments demonstrated that PANDAR expression was induced by p53 protein, and chromatin immunoprecipitation assays confirmed that PANDAR was a direct transcriptional target of p53 protein. In turn, the stability of the p53 protein was markedly reduced by PANDAR silencing. PANDAR stabilizes the p53 protein in response to DNA damage and provides new insight into the regulatory mechanisms of $\mathrm{p} 53^{31,32}$. PANDAR promotes the G1-S transition by suppressing cyclindependent kinase inhibitor p18 or p16 (INK4A) expres$\operatorname{sion}^{33,34}$. In our study, we determined that PANDAR inhibited CDKN1A gene transcription via a competitive interaction with the p53 protein. Mechanically, we identified that PANDAR combined with the p53 protein through its 3 '-terminal sequence of nucleic acids. In turn, the p53 protein interacted with PANDAR via its DNA binding motif. PANDAR inhibited CDKN1A gene transcription by competitively binding to the p53 protein, acting as a p53-response element decoy. In addition, PANDAR controlled its multiple targets mainly by interacting with the $\mathrm{p} 53$ protein.

TP53 functions as a tumour suppressor, and almost half of all human cancers harbour somatic mutations in this gene $^{35}$. There is an urgent need to develop p53-targeting therapy as an approach to treat human cancers. However, p53-targeting therapy alone does not exhibit exciting efficacies in cancer treatment due to the limitation of the adenovirus delivery ${ }^{36}$, the lack of an apoptotic response ${ }^{37}$ and the acquired resistance to p53-targeted therapy ${ }^{38}$. A new opportunity to be investigated is the combination of p53-targeting therapy with conventional chemotherapeutic drugs or other molecularly targeted therapies. Therefore, whether PANDAR depleting combined with a p53 activator might be a benefit for GC therapy was worth investigating. The protein level of p21 in the nude mouse tumour samples was significantly increased in the PANDAR-depletion, nutlin3-treated group, and this combined therapeutic method obviously reduced the nude mouse tumour size and tumour weight in vivo. Additionally, the knockout of PANDAR, combined with nutlin-3, remarkably inhibited the AGS cell lung colonization capacity. These results implied that the therapeutic efficacy of drugs for p53 activation may be strengthened by the RNA interference of lncRNAs.

A significant proportion of studies provide a role for $\mathrm{p} 53$ in the regulation of cell cycle and cell proliferation. Therefore, the identification of IncRNAs regulating p53 function shows a promising potential for the molecular targeting of GC via restoration of p53 activity. Previous studies confirm that the lncRNAs HOTAIR and PANDAR are direct transcriptional targets of p53. Inversely, upregulated HOTAIR inhibits the expression of p53 by enhancing the p53 promoter histone $\mathrm{H} 3$ lysine 27 trimethylation (H3K27me3), and the overexpression of PANDAR increases the stability of the p53 protein in response to DNA damage ${ }^{31,39}$. Here, we present a composite study involving the identification of PANDAR and elucidating its tumour promoting response by competitively binding with p53. PANDAR is a bidirectional transcript from the CDKN1A promoter. The expression of PANDAR and CDKN1A are positively regulated by $\mathrm{p} 53^{40}$. Since CDKN1A mediates cell cycle arrest, PANDAR is able to block apoptosis through its interaction with p53 to limit the expression of CDKN1A and other pro-apoptotic genes. Similar to PANDAR, lincRNA-p21 also serves as a repressor in p53-dependent transcriptional responses by directing the recruitment of hnRNP K to its genomic targets ${ }^{41}$. A number of other lncRNAs are also under direct $\mathrm{p} 53$ regulation or show correlations with p53 levels. The lncRNA MALAT1 is required for G1/S and mitotic progression, and p53 is a major downstream mediator of MALAT1 activity $^{12}$. As a tumour suppressor, MEG3 inhibits tumour cell proliferation possibly through the induction of apoptosis. MEG3 stimulates p53mediated transactivation in human meningioma cell lines $^{42}$. Here, we discuss how lncRNAs serve as p53 regulators or p53 effectors. Further characterization of these p53-associated lncRNAs in cancer will provide a better understanding of IncRNA-mediated gene regulation in the p53 pathway.

In this study, upregulated PANDAR was identified in the GC samples. PANDAR bound to the DNA-binding domain of the p53 protein by acting as a p53-response elements decoy, thus competing with the CDKN1A promoter for binding to the $\mathrm{p} 53$ protein. Through this unique transcriptional modification pattern, PANDAR notably facilitated cancer cell proliferation, clone formation and chemotherapy resistance. Importantly, PANDAR depletion combined with a p53 activator showed perfect efficacy in cancer treatment in vivo. Our results imply that PANDAR is not only a powerful diagnostic biomarker for GC patients but also a promising potential therapeutic target for cancer treatment.

\section{Acknowledgements}

The study was supported by National Natural Science Foundation of China (No. 81772933; No. 81201897; No. 81570948; No. 81472572); Science and Technology Commission of Shanghai Municipality (15QA1402800).

\section{Author details}

'Department of Oncology, Shanghai General Hospital, Shanghai Jiao Tong University School of Medicine, 200080 Shanghai, PR China. ${ }^{2}$ Department of Gastroenterology, Ruijin Hospital, Shanghai Jiao Tong University School of Medicine, 200025 Shanghai, PR China. ³ Department of Stomatology, Renji Hospital, Shanghai Jiao Tong University School of Medicine, 200127 Shanghai, PR China. ${ }^{4}$ Department of Pathology, Fudan University Shanghai Cancer Centre, 200032 Shanghai, PR China. ${ }^{5}$ Department of Integrative Medicine, Zhongshan Hospital, Fudan University, 200032 Shanghai, PR China. 
${ }^{6}$ Department of Oncology, Zhongshan Hospital, Fudan University, 200032 Shanghai, PR China. ${ }^{7}$ Department of Oral and Maxillofacial-Head and Neck Oncology, Ninth People's Hospital, Shanghai Jiao Tong University School of Medicine, 200011 Shanghai, PR China

\section{Conflict of interest}

The authors declare that they have no conflict of interest.

\section{Publisher's note}

Springer Nature remains neutral with regard to jurisdictional claims in published maps and institutional affiliations.

Supplementary Information accompanies this paper at (https://doi.org/ 10.1038/s41419-017-0246-6).

Received: 25 September 2017 Revised: 15 December 2017 Accepted: 18 December 2017

Published online: 07 February 2018

\section{References}

1. Ferro, A. et al. Worldwide trends in gastric cancer mortality (1980-2011), with predictions to 2015, and incidence by subtype. Eur. J. Cancer 50, 1330-1344 (2014).

2. Catalano, V. et al. Gastric cancer. Crit. Rev. Oncol. Hematol. 71, 127-164 (2009).

3. Dassen, A. E. et al. Changes in treatment patterns and their influence on longterm survival in patients with stages I-III gastric cancer in The Netherlands. Int. J. Cancer 133, 1859-1866 (2013).

4. Nadauld, L. D. et al. Metastatic tumor evolution and organoid modeling implicate TGFBR2 as a cancer driver in diffuse gastric cancer. Genome Biol. 15, 428 (2014).

5. Ponting, C. P., Oliver, P. L. \& Reik, W. Evolution and functions of long noncoding RNAs. Cell 136, 629-641 (2009).

6. Imamura, K. \& Akimitsu, N. Long non-coding RNAs involved in immune responses. Front. Immunol. 5, 573 (2014)

7. Amaral, P. P., Dinger, M. E., Mercer, T. R. \& Mattick, J. S. The eukaryotic genome as an RNA machine. Science 319, 1787-1789 (2008).

8. Li, L. et al. Role of human noncoding RNAs in the control of tumorigenesis. Proc. Natl. Acad. Sci. USA 106, 12956-12961 (2009).

9. Gupta, R. A. et al. Long non-coding RNA HOTAIR reprograms chromatin state to promote cancer metastasis. Nature 464, 1071-1076 (2010).

10. Bao, X. et al. Knockdown of long non-coding RNA HOTAIR increases miR-454$3 p$ by targeting Stat3 and Atg12 to inhibit chondrosarcoma growth. Cell Death Dis. 8, e2605 (2017).

11. Hong, Q. et al. LnCRNA HOTAIR regulates HIF-1alpha/AXL signaling through inhibition of miR-217 in renal cell carcinoma. Cell Death Dis. 8, e2772 (2017).

12. Tripathi, V. et al. Long noncoding RNA MALAT1 controls cell cycle progression by regulating the expression of oncogenic transcription factor B-MYB. PLOS Genet. 9, e1003368 (2013).

13. Endo, $\mathrm{H}$. et al. Enhanced expression of long non-coding RNA HOTAIR is associated with the development of gastric cancer. PLOS ONE 8, e77070 (2013).

14. $\mathrm{Li}, \mathrm{H}$. et al. Overexpression of IncRNA H19 enhances carcinogenesis and metastasis of gastric cancer. Oncotarget 5, 2318-2329 (2014).

15. Hu, Y. et al. Long noncoding RNA GAPLINC regulates CD44-dependent cell invasiveness and associates with poor prognosis of gastric cancer. Cancer Res. 74, 6890-6902 (2014).

16. Sun, M. et al. Decreased expression of long noncoding RNA GAS5 indicates a poor prognosis and promotes cell proliferation in gastric cancer. Bmc Cancer 14, 319 (2014).

17. Xia, T. et al. Long noncoding RNA associated-competing endogenous RNAs in gastric cancer. Sci. Rep. 4, 6088 (2014).
18. Han, Y. et al. LEIGC long non-coding RNA acts as a tumor suppressor in gastric carcinoma by inhibiting the epithelial-to-mesenchymal transition. Bmc. Cancer 14, 932 (2014).

19. Zhang, J. et al. Transcriptional control of PAX4-regulated miR-144/451 modulates metastasis by suppressing ADAMs expression. Oncogene 34, 3283-3295 (2015).

20. Guo, $H$. et al. The aspirin-induced long non-coding RNA OLA1P2 blocks phosphorylated STAT3 homodimer formation. Genome Biol. 17, 24 (2016).

21. $\mathrm{Xu}, \mathrm{Y}$., Jiang, $\mathrm{X}$. \& Cui, Y. Upregulated long noncoding RNA PANDAR predicts an unfavorable prognosis and promotes tumorigenesis in cholangiocarcinoma. OncoTargets Ther. 10, 2873-2883 (2017).

22. Zhan, Y. et al. Up-regulation of long non-coding RNA PANDAR is associated with poor prognosis and promotes tumorigenesis in bladder cancer. J. Exp. Clin. Cancer Res. 35, 83 (2016).

23. $\mathrm{Xu}, \mathrm{Y}$. et al. An increase in long non-coding RNA PANDAR is associated with poor prognosis in clear cell renal cell carcinoma. Bmc Cancer 17, 373 (2017).

24. Peng, W. \& Fan, H. Long non-coding RNA PANDAR correlates with poor prognosis and promotes tumorigenesis in hepatocellular carcinoma. Biomed. Pharmacother. 72, 113-118 (2015).

25. Lu, M. et al. The high expression of long non-coding RNA PANDAR indicates a poor prognosis for colorectal cancer and promotes metastasis by EMT pathway. J. Cancer Res. Clin. Oncol. 143, 71-81 (2017).

26. Ma, P., Xu, T., Huang, M. \& Shu, Y. Increased expression of LncRNA PANDAR predicts a poor prognosis in gastric cancer. Biomed. Pharmacother. 78, 172-176 (2016)

27. Khalil, A. M. et al. Many human large intergenic noncoding RNAs associate with chromatin-modifying complexes and affect gene expression. Proc. Natl. Acad. Sci. USA 106, 11667-11672 (2009).

28. Yang, $F$. et al. The IncRNA Firre anchors the inactive $X$ chromosome to the nucleolus by binding CTCF and maintains H3K27me3 methylation. Genome Biol. 16, 52 (2015).

29. Martianov, I., Ramadass, A., Serra Barros, A., Chow, N. \& Akoulitchev, A. Repression of the human dihydrofolate reductase gene by a non-coding interfering transcript. Nature 445, 666-670 (2007).

30. Lopez-Pajares, V. et al. A LnCRNA-MAF:MAFB transcription factor network regulates epidermal differentiation. Dev. Cell 32, 693-706 (2015).

31. Han, L. et al. Low expression of long noncoding RNA PANDAR predicts a poor prognosis of non-small cell lung cancer and affects cell apoptosis by regulating BCl-2. Cell Death Dis. 6, e1665 (2015).

32. Kotake, Y. et al. Long non-coding RNA, PANDA, contributes to the stabilization of p53 tumor suppressor protein. Anticancer Res. 36, 1605-1611 (2016).

33. Kotake, Y. et al. Long noncoding RNA PANDA positively regulates proliferation of osteosarcoma cells. Anticancer Res. 37, 81-85 (2017).

34. Sang, $Y$. et al. LncRNA PANDAR regulates the G1/S transition of breast cancer cells by suppressing p16(INK4A) expression. Sci. Rep. 6, 22366 (2016).

35. Berns, A. Cancer: the blind spot of p53. Nature 468, 519-520 (2010).

36. Zeimet, A. G. \& Marth, C. Why did p53 gene therapy fail in ovarian cancer? Lancet Oncol. 4, 415-422 (2003).

37. Ventura, $A$. et al. Restoration of p53 function leads to tumour regression in vivo. Nature 445, 661-665 (2007).

38. Martins, C. P., Brown-Swigart, L. \& Evan, G. I. Modeling the therapeutic efficacy of p53 restoration in tumors. Cell 127, 1323-1334 (2006).

39. Zhai, N., Xia, Y., Yin, R., Liu, J. \& Gao, F. A negative regulation loop of long noncoding RNA HOTAIR and p53 in non-small-cell lung cancer. OncoTargets Ther. 9, 5713-5720 (2016).

40. Hung, T. et al. Extensive and coordinated transcription of noncoding RNAs within cell-cycle promoters. Nat. Genet. 43, 621-629 (2011).

41. Yoon, J. H. et al. LincRNA-p21 suppresses target mRNA translation. Mol. Cell 47, 648-655 (2012).

42. Zhang, $X$. et al. Maternally expressed gene 3 (MEG3) noncoding ribonucleic acid: isoform structure, expression, and functions. Endocrinology 151, 939-947 (2010). 